Running head: SLEEP AND STRESS ON RISKY DECISIONS

Sleep Duration Moderates the Association Between Insula Activation and Risky Decisions Under Stress in Adolescents and Adults

Jessica Phuong $\mathrm{Uy}^{1} \&$ Adriana Galván ${ }^{1 *}$

${ }^{1}$ Department of Psychology University of California, Los Angeles

*Corresponding Author

Adriana Galván, $\mathrm{PhD}$

1285 Franz Hall, Box 951563

University of California, Los Angeles,

Los Angeles, CA 90095

tel:(310)206-4850

Acknowledgements: This research was funded by the Jacobs Foundation and National Science Foundation (BCS 0963750) to AG. 


\begin{abstract}
Insufficient sleep has been associated with increased risk-taking and poor decision-making, enhanced physiological responses to stress, and attenuated anterior insula (AI) activity to risk. The AI has also been linked to risky decision-making under acute stress. However, it is yet unknown how naturalistic sleep habits affect risky decision-making and AI activity when individuals feel stressed. In the current study, a daily diary approach was used to document participants’ daily stress. Adolescents and adults reported their recent sleep duration and completed two fMRI visits during which they performed a risky decision-making task: once each when they endorsed a high and low level of stress. Results revealed that, regardless of age, individuals who reported receiving more sleep took fewer non-advantageous risks during high stress relative to those who reported receiving fewer hours of sleep per night while sleep duration was not associated with risky behavior under low stress. Among individuals who reported less sleep, those who exhibited reduced AI activation during risk-taking under high stress also took more disadvantageous risks whereas this effect was attenuated for those who reported longer sleep duration. Moreover, longer sleep duration was associated with greater functional coupling between the AI and dorsolateral prefrontal cortex (DLPFC) under high stress whereas sleep duration was not associated with AI-DLPFC functional coupling under low stress. These findings suggest that naturalistic sleep duration may amplify the effects of daily stress and alter risky decision-making behavior through interactions with the AI.
\end{abstract}

Keywords: stress, sleep, risky decision, insula 


\section{Sleep Duration Moderates the Association Between Insula Activation and Risky Decisions Under Stress in Adolescents and Adults}

The National Sleep Foundation recommends that adults and adolescents attain 7-9 hours and 8-10 hours of sleep per night, respectively (Hirshkowitz et al., 2015). However, according to the Centers for Disease Control and Prevention (CDC), about 35\% of adults and over 60\% of high school students receive less than the recommended duration of sleep(CDC, 2011; Kann et al., 2014). Individuals who receive poor sleep are at an increased risk for physical and psychological health problems(Vgontzas et al., 2004) and exhibit deficits in learning and memory(Walker \& Stickgold, 2006) and behavioral inhibition(Anderson \& Platten, 2011; Telzer, Fuligni, Lieberman, \& Galván, 2013). Neurocognitive deficits give rise to increased risk-taking and poor decision-making(Harrison \& Horne, 2000; Killgore, Balkin, \& Wesensten, 2006; Killgore, Kamimori, \& Balkin, 2011).Extensive research in adults has shown that sleep loss leads to similar biological outcomes as those induced by stress and amplifies physiological responses to stress(Balbo, Leproult, \& Van Cauter, 2010; Spiegel, Leproult, \& Van Cauter, 1999; Vgontzas et al., 2004). For example, sleep-deprived adults have higher baseline cortisol levels and increased cortisol response to psychosocial stress relative to well-rested individuals (Minkel et al., 2014); this physiological response to stress appears to occur via enhanced limbic reactivity and diminished amygdala-prefrontal connectivity(Anderson \& Platten, 2011; Yoo, Gujar, Hu, Jolesz, \& Walker, 2007). It is likely that sleep loss may also amplify the effects of acute stress on risky decision-making. Given how often individuals generally experience stress, how prevalent insufficient sleep is, and how many decisions individuals are required to make daily under these conditions, it is surprising that these relationships have never been examined 
together in adults or in adolescents. The goal of the current study was to investigate how average sleep duration might moderate the relationship between daily stress and risky decisions.

In adults, acute stress alters neural activation of mesolimbic-prefrontal circuitry, including the striatum, anterior insula (AI), and prefrontal (PFC) regions(Kogler et al., 2013; Pruessner et al., 2008)through cortisol-related increases of dopamine release in the ventral striatum and PFC(Pruessner, Champagne, Meaney, \& Dagher, 2004; Ungless, Argilli, \& Bonci, 2010). These neural changes enhance reward salience and lead to greater reward-biased decisions coupled with decreased sensitivity to punishment and altered strategy use during decision-making(Porcelli \& Delgado, 2009; Putman, Antypa, Crysovergi, \& van der Does, 2010; Starcke \& Brand, 2012; Starcke, Wolf, Markowitsch, \& Brand, 2008). For example, stressed individuals who exhibited greater increases in cortisol chose options that could potentially lead to high reward but actually led to high punishment more often(Starcke et al., 2008). Another study reported that participants under acute stress took more risks in a loss domain versus gain domain(Porcelli \& Delgado, 2009), suggesting that stress may decrease sensitivity to punishment.Using risky decision-making tasks in functional magnetic resonance imaging (fMRI), Lighthall et al. (2012) found that acute stress enhanceddorsal striatum and AI activation during decision-making in adult males. AI activation was positively correlated with reward collection rate, which was one of the adaptive strategies that optimized earnings(Lighthall et al., 2012), suggesting that the AI may play a role in adaptive risk-taking under stress.

Research examining the effects of acute stress on risk-taking in adolescents has been limited. Previous work from our lab examined how daily stress affects adaptive risky decisionmaking in adolescents and adults (Uy \& Galván, 2016). Using a within-subjects daily diary approach to measuring daily stress and an adaptive risky decision-making task, we showed that 
adolescent males (15-17 years) took more advantageous risks under high relative to low stress whereas adult males (25-30 years) took fewer non-advantageous (equal expected value) risks under high relative to low stress. Moreover, despite showing similar sensitivity to expected value, adolescent males demonstrated increased overall risk preference under high stress relative to adult males while there were no differences in risky decisions under low stress conditions(Uy \& Galván, 2016). These developmental differences in stress-related risky behaviors were paralleled by developmental differences in stress-related functional activation in the PFC during adaptive risky decision-makingsuch that adolescent males evinced reduced PFC activation during high relative to low stress when making advantageous decisions (relative to disadvantageous decisions) whereas adult males showed no stress-related changes in PFC recruitment(Uy \& Galván, 2016). Moreover, decreasing PFC activation under stress was related to greater non-advantageous risks under stress, suggesting that the developing PFC may be more sensitive to the effects of stress.

fMRI studies investigating the effects of sleep deprivation on financial risky decisionmaking in adults have shown that sleep deprivation is associated with increased risk-taking for gains and decreased loss-avoidance behavior as a result of diminished use of expected value information to maximize expected outcomes(Libedinsky et al., 2011; Menz, Buchel, \& Peters, 2012; Mullette-Gillman, Kurnianingsih, \& Liu, 2015). Individuals who were sleep deprived also showed reduced AI activation and increased ventromedial prefrontal cortex (vmPFC) activation when making decisions about losses and gains, respectively, compared to when they were well rested(Venkatraman, Chuah, Huettel, \& Chee, 2007; Venkatraman, Huettel, Chuah, Payne, \& Chee, 2011). Decreasing AI activation was related to less loss-minimizing behavior while increasing vmPFC activation increased gain-maximizing behavior (Venkatraman et al., 2011). In 
other words, sleep loss appears to bias individuals to be more motivated to seek positive outcomes than to avoid negative outcomes.

The AI integrates affective and cognitive states(Bechara, Damasio, Tranel, \& Damasio, 1997; Bechara \& Damasio, 2005; Werner et al., 2013) and is involved in emotional decisionmaking and reward expectation(Mizuhiki, Richmond, \& Shidara, 2012). It is sensitive to expected value(Droutman, Bechara, \& Read, 2015; Weller, Levin, Shiv, \& Bechara, 2009) and plays a critical role in adaptive decision-making(Preuschoff, Quartz, \& Bossaerts, 2008; Xue, Lu, Levin, \& Bechara, 2011). During adolescence, the AI undergoes ongoing maturation (Shaw et al., 2008; Smith, Steinberg, \& Chein, 2014). The connectivity strength between the AI and PFC continues to increase with age (Fair et al., 2007)whereas connectivity between the AI, striatum, and anterior cingulate cortex (ACC) reaches adult-like strength by adolescence (Stevens, Pearlson, \& Calhoun, 2009). These developmental differences contribute to the underlying immature cognitive control and heightened reward sensitivity in adolescents relative to adults, biasing them towards risky decisions. Indeed, on a risky decision-making task, adolescents exhibited reduced AI activation compared to adults during risk-taking, which was thought to reflect immature integration of affective and cognitive information related to the consequences of their decisions (Eshel, Nelson, Blair, Pine, \& Ernst, 2007). Additionally, in adolescents (14-16 years), Telzer et al.(2013)found that poor sleep quality was associated withreduced functional coupling between the AI and dorsolateral PFC (DLPFC) during reward processing, which predicted greater risk-taking. These findings suggest that, in addition to functional activation of the AI alone, stronger functional coupling between cognitive and affective systems via the AI might underlie more mature/rational risk-taking behavior, and that 
thestrength of functional coupling between these systems in the context of risk can be influenced by sleep.

Taken together, evidence suggests that poor sleep and acute stress may alter risky decision-making by undermining optimal regulation of reward-biased risk-taking behavior through modifications of the insular cortex, prefrontal cortex, and/or functional coupling between the two regions in both adolescents and adults. Given that sleep loss and acute stress act on similar neural and physiological systems to alter risky decision-making and that these systems under significant maturation during adolescence, it is important to investigate how poor sleep might interact with daily stress to amplify the effects of acute stress on risky decision-making in both adolescents and adults.

The current study aimed to determine how naturalistic sleep loss (via self-reported sleep duration) affects risky decision-making and AI activity when individuals feel stressed (relative to when they do not feel stressed). Given the differential effects of sleep loss and stress on gains versus losses as well as advantageous versus non-advantageous risky decision-making, participants performed an adaptive risky decision-making task (the Cups Task) that differentiated between these types of risky choices. We were also interested in examining whether sleep duration was related to self-reported perceptions of riskiness, perceived benefits of risks, and the likelihood to take risks in the real world. Based on previous research, we made the following predictions: 1) individuals who reported receiving fewer hours of sleep per night would take more non-advantageous risks (perhaps more so in the context of potential gains) under high stress while sleep duration may not affect risk-taking under low stress (i.e., sleep x stress interaction); 2) individuals would activate the AI when taking risks and those who exhibited reduced AI activation during risk-taking under high stress would take more non-advantageous 
risks; 3) the relationship between AI activation and non-advantageous risks under stress would be more pronounced in individuals who reported shorter sleep relative to longer sleep; 4) we predicted that the interaction between sleep duration and daily stress on non-advantageous risktaking would be exaggerated in adolescents relative to adults (i.e., an age x sleep x stress interaction).Regarding real-world risk-taking, we predicted that individuals who reported fewer hours of sleep would report greater perceived benefits from a number of risks and greater likelihood to take risks in the real world. Previous studies have found gender differences in the effects of acute stress on risky decision-making (e.g., Lighthall et al., 2012; Lighthall, Mather, \& Gorlick, 2009; Putman et al., 2010; Uy \& Galván, 2016), and the findings thusfar have been rather inconsistent. We do not have any specific predictions regarding gender; therefore, gender was included as a covariate in the current study.

\section{Method}

\subsection{Participants}

Twenty-two adolescents (14 females) between $15-17$ years of age $(M=16.55, S D=.67)$ and twenty-two adults (12 females) between 25-30 years of age $(M=27.27, S D=1.72)$ participated in the study. Participants were recruited through advertisements on the UCLA campus, surrounding neighborhoods, and local online classifieds and forums. Participants were excluded if they had any metal objects in their bodies (e.g., braces, permanent retainers), a diagnosis of a psychiatric or developmental disorder, claustrophobia, were left-handed, or were pregnant. Informed consent was obtained from all adult participants; parental consent and assent was obtained from all participants under the age of 18 in accordance with procedures approved by the UCLA Institutional Review Board. The Wechsler Abbreviated Scale of Intelligence (WASI) was administered to estimate IQ. Adolescents and adults did not significantly differ in 
IQ $(t(42)=-.868, p=.390)$ or ethnic composition $\left(X^{2}(5, \mathrm{~N}=44)=5.524, p=.355\right)$.

Socioeconomic status (SES) was determined by participant's maternal level of education. Recent stressful life events (SLE) was determined by participant's self-reported number of stressful life experiences in the past 6 months on a Life Events checklist (e.g., death of close family member, begin new school, change in living conditions, breakup with significant other, etc.). Adolescents and adults did not significantly differ in $\operatorname{SES}\left(X^{2}(6, \mathrm{~N}=44)=6.210, p=.400\right)$, or in the number of recent SLE, $(t(42)=.411, p=.683)$.

\subsection{Materials}

\subsubsection{Sleep Patterns Questionnaire}

Sleep duration was determined by participants’ self-reported response to the following question during the first visit of the study: "On average, how many hours have you slept per night in the past month?” This question serves to capture a naturalistic depiction of individuals’ recent habitual sleep duration and allows us to investigate how this average "baseline” sleep duration reported at the time of study enrollment might influence subsequent reactivity to stress. Self-reported sleep duration has been shown to be moderately correlated $(r=0.45)$ with objective sleep measures obtained using actigraph watches (Biddle, Robillard, Hermens, Hickie, \& Glozier, 2015; Cespedes et al., 2016; Lauderdale, 2008), making this measure a suitable measure of recent habitual sleep duration. Furthermore, self-reports of sleep duration via questionnaire have also been shown to be moderately reliable with both daily diary $(r=0.61)$ and actigraph $(r$ = 0.53) methods in adolescents(Short, Gradisar, Lack, Wright, \& Carskadon, 2012; Wolfson et al., 2003). Sleep duration ranged from 5-10 hours per night $(M=6.84, S D=1.26)$. Adolescents reported sleeping an average of 6.75 hours per night $(S D=1.45)$. Adults reported sleeping an average of 6.93 hours per night $(S D=1.08)$. There were no significant differences in sleep 
duration among age $(p=.641)$ and gender groups $(p=.757)$. However, because of the difference in NSF-recommended sleep duration, this would imply that more adolescents are sleeping less than their recommended 8-hour minimum $(n=15)$ than adults are sleeping less than their recommended 7-hour minimum $(n=9)$. A Chi-square test revealed no significant differences in the distribution of individuals who reported receiving insufficient sleep between adolescents and adults $\left(X^{2}(1, \mathrm{~N}=44)=3.30, p=.069\right)$.

\subsubsection{Domain-Specific Risk-Taking Scale}

Participants completed the Domain-Specific Risk-Taking Scale (DOSPERT; Figner \& Weber, 2012), a well-validated 40-item measure of one's perceived risk of, expected benefit of, and likelihood of engaging in risky events in the following domains: ethical, financial, health/safety, recreational, and social. Age-appropriate versions of the DOSPERT for adults and adolescents (ages 14-17) were administered based on participant age. For example, the adolescent version of the DOSPERT investigates ethical risk-taking by asking participants to consider “dating someone else's boyfriend or girlfriend” while adults are asked to consider “having an affair with a married man or woman.” The DOSPERT uses a 7-point Likert scale for each subscale ("not at all risky” to "extremely risky” for perceived risk, "no benefits at all” to “great benefits” for expected benefits, and “extremely unlikely” to “extremely likely” for likelihood of risk engagement). Self-reported ratings on each subscale were averaged across domains to create mean composite scores for perceived risk, expected benefit, and likelihood of engaging in risky events.

\subsubsection{Cups Risky Decision-Making Task}

The Cups Task(Levin \& Hart, 2003; Levin, Weller, Pederson, \& Harshman, 2007) measures decision-making under uncertainty, as individuals often make decisions under 
uncertain conditions. The Cups Task has been used in previous fMRI studies to study the neural correlates of risky decision-making (Xue et al., 2009; Xue, Lu, Levin, \& Bechara, 2013). Participants were presented with two task frames (Frame): a frame where they had the opportunity to gain money (Gain Frame, $n=54$ trials), and one where they could lose money (Lose Frame, $n=54$ trials). Depending on the frame, participants were asked to choose between a certain gain (or loss) and an uncertain gain (or loss). The neutral terms "certain” and "uncertain” were used during explanation of the task to participants to prevent suggestion of any connotations or biases that might be associated with the terms "safe” and "risky", respectively, which are used throughout the report. The certain option was to win (or lose) \$2, while the uncertain option led to a probability (20\%, 33\%, or $50 \%$ ) of either a larger win (or loss) (\$4, $\$ 6$, or $\$ 10$ ) or no win (or loss). The certain option consisted of one flipped-over cup with \$2 written underneath it, indicating to participants that there is a $100 \%$ chance of gaining (or losing) \$2. In the uncertain option, the number of cups varied between two (50\%), three (33\%), and five (20\%) cups, and the amount indicated underneath each set of cups varied between $\$ 4, \$ 6$, and $\$ 10$. There were three trial types $(\mathrm{n}=36 /$ trial type) that differed in expected value $(\mathrm{EV}=$ value $\mathrm{x}$ probability). The advantageous EV trials (ADV), in which the EV was greater than $\$ 2$ in the gain frame or less than $\$ 2$ in the lose frame, consisted of the $50 \%-\$ 6,50 \%-\$ 10$, and $33 \%-\$ 10$ trials in the gain frame and 33\%-\$4, 20\%-\$4, and 20\%-\$6 trials in the lose frame; in these trials, the uncertain choice yielded better EV than the certain choice. The disadvantageous EV trials (DIS), in which the EV was less than \$2 in the gain frame and greater than \$2 in the lose frame, consisted of the $33 \%-\$ 4,20 \%-\$ 4$, and $20 \%$ - $\$ 6$ trials in the gain frame and the $50 \%-\$ 6,50 \%$ $\$ 10$, and 33\%-\$10 trials in the lose frame; in these trials, the uncertain choice yielded worse EV than the certain choice. In the equal EV trials (EQEV), in which the EV was \$2, consisted of the 
50\%-\$4, 33\%-\$6, and 20\%-\$10 trials in both gain and lose frames; in these trials, the uncertain choice yielded the same EV as the certain choice. Participants were instructed to consider each choice carefully and to earn as much money as possible in the game because at the end of the game, the computer would randomly select an outcome based on their decisions and that outcome would be added to or subtracted from their study compensation. Participants did not actually lose any money; this information was not disclosed to participants until after completion of the study. Thus, participants could earn between $\$ 2$ and $\$ 10$ in addition to study compensation. All participants completed two 7-minute runs $(\mathrm{n}=108$ total trials) of the task during each stress visit for a total of four 7-minute runs across both stress visits ( $\mathrm{n}=216$ total trials) (Figure 1).

The percentage of risks taken on the Cups Task for each trial type was calculated by counting the number of times participants chose the risky option of each trial type divided by the total number of trials presented of each trial type, multiplied by 100 . The trial types were as followed: Gain ADV (ADV trials in Gain frame), Gain EQEV (EQEV trials in Gain frame), Gain DIS (DIS trials in Gain frame), Lose ADV (ADV trials in Lose frame), Lose EQEV (EQEV trials in Lose frame), Lose DIS (DIS risks in Lose frame). Percentages of risks taken for each trial type were calculated for each stress visit and were used as dependent variables in data analysis. 


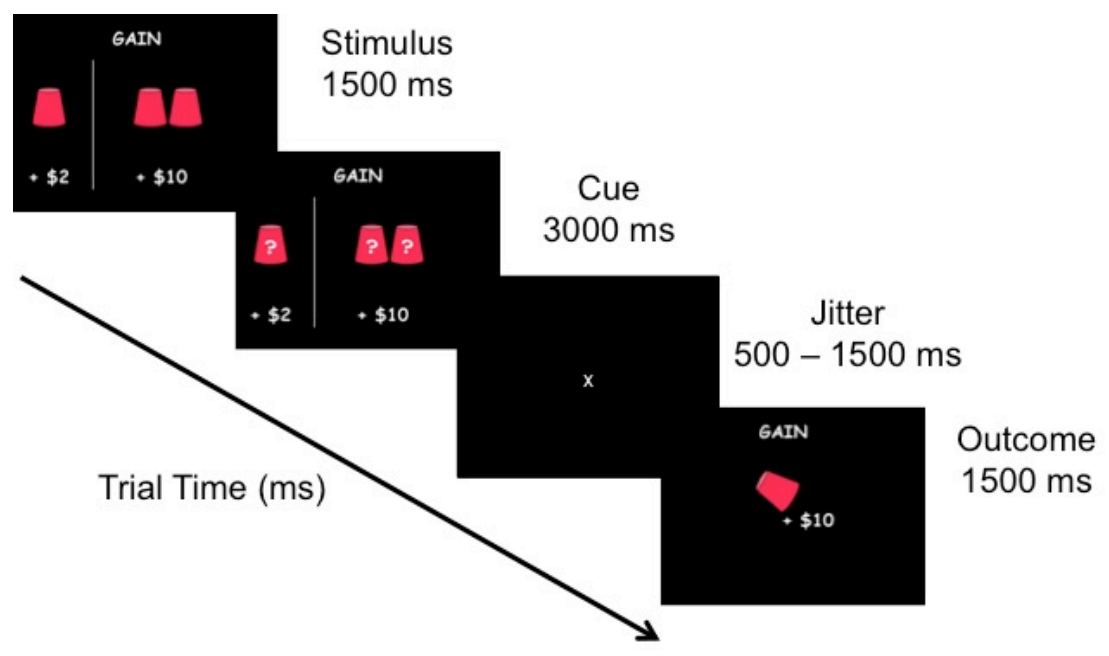

Figure 1. Schematic of Cups Task. Each stimulus was presented for 1500 ms. After stimulus presentation, participants are asked to choose between the certain (left) side or the uncertain (right) side (Cue). Once participants made their decision, a jittered inter-stimulus-interval (ISI) was shown for 500-1500ms followed by outcome presentation (Outcome) for $1500 \mathrm{~ms}$.

\subsection{Procedure}

Each participant was enrolled in the study for two weeks. After providing consent, participants completed questionnaires and received instructions regarding the study procedures. Participants provided salivary cortisol samples at the beginning and end of the intake visit to assess baseline physiological stress response to the novel, laboratory setting. Salivary cortisol is a commonly used indicator of an organism's response to stress and reflects activity of the hypothalamic-pituitary-adrenal (HPA) axis(Hellhammer, Wüst, \& Kudielka, 2009).

\subsubsection{Daily stress assessment}

Daily stress was assessed using an ecological momentary assessment (EMA; Bolger, Davis, \& Rafaeli, 2003)method, a procedure in which participants were contacted daily through smartphones and reported daily ratings of subjective feelings of stress. A smartphone was 
available from the lab to participants who did not have a cellular phone and/or a text-messaging plan. However, all participants in the study used their personal cellular phones. The EMA method has been shown to be successful at capturing naturally occurring stress (Almeida, McGonagle, \& King, 2009; Galván \& Rahdar, 2013; Galván \& McGlennen, 2012). In the current study, participants were randomly text-messaged three times per day between the hours of 9:00

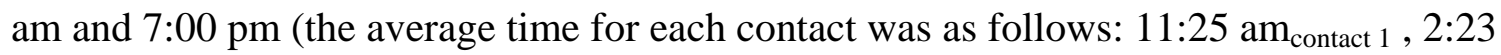
$\mathrm{pm}_{\text {contact2 }}, 5: 00 \mathrm{pm}_{\text {contact 3) }}$ over the span of 2 weeks and were asked to indicate the level of stress they experienced in the last hour using a Likert scale ( 1 = not stressed; 7 = very stressed).

\subsubsection{Baseline assessment}

During the first three days, each participant's baseline composite stress rating was determined by averaging the stress ratings provided via text messages throughout the day across the three days (average baseline stress rating $=3.02, S D=.90$ ). Concurrently, during the first two consecutive days, participants collected salivary cortisol via passive droolat home (releasing saliva into tube through a straw) to evaluate each participant's baseline diurnal cortisol pattern. Participants were instructed not to eat or drink anything at least 30 minutes prior to collection and to collect $1 \mathrm{~mL}$ of saliva at awakening, 30 minutes post-awakening, at 4:30pm, and at 8:30pm using Salivette ${ }^{\circledR}$ test tubes (Sarstedt, Germany) and to store the samples in their freezers until their next scheduled laboratory visit. The average time between participants’ first saliva collection and when participants returned samples was 5.84 days $(S D=2.53)$. This method and timing of salivary cortisol collection has been shown to be standard and reliable (Clements, 2012). All participants returned completed sets of saliva samples. All samples were stored at $20^{\circ} \mathrm{C}$ in a laboratory freezer at UCLA until time of shipment. Salivary cortisol analyses were conducted as described byStrahler, Mueller, Rosenloecher, Kirschbaum, and Rohleder(2010). 


\subsubsection{Stress visits}

Two subsequent laboratory visits were categorized into high-stress and low-stress visits based on stress ratings relative to baseline stress ratings. High-stress state was determined when participants endorsed stress ratings that were at least one standard deviation above their baseline stress rating (or a 1-point increase in stress rating if baseline rating was relatively high (i.e., 5 or greater; $\mathrm{n}=0$ participants)).Similarly, low-stress state was determined when participants endorsed stress ratings that were at least one standard deviation below their baseline stress rating (or a 1-point decrease in stress rating if baseline rating was relatively low (i.e., 2 or lower; $\mathrm{n}=4$ adolescents, 2 adults)). After a high-stress state or low-stress state was determined, participants were asked to visit the laboratory in the late afternoon of the same day. Since the stress conditions were driven by participant responses, true experimenter-controlled counterbalancing of whether participants’ first scan was for low or high stress was not possible in this study design. However, due to the random sampling of the days and participants, it turned out that exactly 50\% ( $n=22)$ of our participants (and 50\% from each age group $[n=11])$ completed their low stress scan before their high stress scan while the other $50 \%$ completed their high stress scan before their low stress scan. Scan order was included as a covariate in all behavioral and fMRI analyses.

The average duration between the start of the study and the first scan was 5.84 days (SD $=2.53)$. The average duration between the first scan and the second scan was 6.27 days $(S D=$ 2.11). There were no significant differences in duration between adolescents and adults ( $p>.4)$. At each stress visit, salivary cortisol was collected before participants underwent an fMRI scan to assess physiological response to stress as close in time to stressor onset as possible. Duration between reported stress (stressor) and brain scan ranged from 30 minutes to 5 hours and 30 
minutes ( $M=2$ hours and 7 minutes, $S D=1$ hour, 6 minutes). Duration did not significantly differ by stress state $(p=.263)$, age group $(p=.729)$, or gender $(p=.555)$. Average stress rating for high stress reported via text-message was $4.20(S D=1.53)$ while average rating for low stress was $2.20(S D=1.18)$. Participants also reported stress ratings when they arrived for the fMRI scan. Average stress rating upon arrival was $4.42(S D=1.44)$ for high stress and $2.47(S D=$ 1.27) for low stress scan. There were no significant differences between ratings reported at text and those reported upon arrival for the scan, which suggests that the stress state was sustained. Stress ratings reported upon arrival for the scan were used for analyses, as they were closer in time to the scan and may reflect a more accurate assessment of participants' stress state at the time of the scan. During each visit, participants underwent an fMRI scan while performing the Cups Task.

\subsection{MRI Data Acquisition}

Functional imaging data were collected on a 3 Tesla Siemens Trio MRI scanner using a gradient-echo, echo-planar image (EPI) sequence (TR: 2 s, TE: 30 ms, flip angle: 90 271 volumes, 34 slices, slice thickness: 4 mm). A T2-weighted, matched bandwidth (MBW), highresolution anatomical scan and magnetization-prepared rapid-acquisition gradient echo (MPRAGE) scan were acquired for registration purposes (TR: 2.3, TE: 2.1, FOV: 256, matrix: 192 x 192, sagittal plane, slice thickness: $1 \mathrm{~mm}, 160$ slices). The orientation for the MBW and EPI scans was oblique axial to maximize brain coverage. E-prime software was used for task presentation.

\subsection{Image Preprocessing and Registration}

Preprocessing and statistical analyses were performed using FMRIB’s Software Library (FSL) 5.0.9 (www.fmrib.ox.ac.uk/fsl). Preprocessing included motion correction, non-brain 
matter removal using FSL brain extraction tool (BET), spatial smoothing (5mm FWHM Gaussian kernel) to increase the signal-to-noise ratio, and filtered in the temporal domain using a nonlinear high-pass filter (100-s cutoff). All data reported are from scans with $\leq 2 \mathrm{~mm}$ in translational movement. EPI images were registered to the MBW scan, then to the MPRAGE scan, and finally into standard Montreal Neurological Institute (MNI) space (MNI152, T1 2mm) using linear registration with FSL FMRIB’s Linear Image Registration Tool (FLIRT).

\subsection{Functional Neuroimaging Data Analysis}

One general linear model (GLM) with multiple regressors (stimulus presentation, decision response, and outcome) was used for fMRI analyses. Regressors were created by convolving a delta function representing the onset time of each event with a canonical (double gamma) HRF. Onset time for the stimulus presentation regressor was defined as the onset of the stimulus for each trial with no duration. Onset time for decision response event was defined as the onset of the cue presentation for each trial with reaction time as the duration. Onset time for outcome event was defined as the onset of the outcome presentation of each trial with no duration. For each participant, each stress visit, and each run, five explanatory variables were modeled: 1) all stimuli presentations; 2) all risky (uncertain) choices; 3) all safe (certain) choices; 4) all outcome presentations, and 5) missed/invalid trials. Six motion parameters were modeled as events of no interest in the GLM. Temporal derivatives were included as covariates of no interest. Inter-trial and inter-stimulus interval fixation points were not explicitly modeled and therefore served as an implicit baseline.

fMRI analyses focused on participant choice (risky or safe) for high stress scans and low stress scans. To examine the effects of risk-taking, the following contrasts were modeled for each run: 1) risky choice (relative to implicit baseline); 2) safe choice (relative to implicit baseline); 3) 
risky choice > safe choice; and 4) safe choice > risky choice. A fixed effects voxel-wise analysis combined each of two runs for each stress visit for each participant at the second level.

2 (Stress) x 2 (Age) x 2 (Gender) repeated measures analyses of variance (ANOVA) were conducted at the group level using the FMRIB local analysis of mixed effects (FLAME1) module in FSL with and without sleep duration (mean-centered) entered as a regressor in the model. Given our a priori hypotheses regarding the role of the anterior insula, a region-ofinterest (ROI) mask for bilateral anterior insula was anatomically localized and defined using masks from FSL's Harvard-Oxford atlas. Z (Gaussianized T) statistic images were threshold using the ROI mask, determined by $\mathrm{Z}>2.3$ and a corrected cluster significant threshold of $\mathrm{p}<$ .05 using Gaussian Random Field theory. Tests were corrected for family-wise errors (FWE).

\subsection{Psychophysiological Interaction (PPI) Analyses}

Psychophysiological interaction (PPI) analyses(Friston et al., 1997) were conducted to examine whether functional coupling between the anterior insula and subcortical and prefrontal regions was 1) affected by stress, 2) modulated by sleep duration, and/or 3) predicted risky behavior in adolescents and adults. The seed region for the PPI analyses was defined as the area in the anterior insula that was activated during risk-taking and was affected by stress. The seed region was first transformed into the functional space of each participant using FLIRT in FSL, and the deconvolved time-series was extracted for the ROI. The first-level design for each run consisted of three regressors of interest: 1) the physiological regressor, 2) the psychological variable, and 3) their product. The physiological regressor comprised the time-series for the anterior insula. The psychological (task)regressor modeled the contrast of risky choice versus safe choice, convolved with a double-gamma hemodynamic response function (HRF). The product regressor modeled the interaction between of the psychological regressor and the 
physiological regressor, with the psychological regressor zero-centered about the minimum and maximum values and the physiological regressor demeaned. This interaction term identified regions that covaried in a task-dependent manner with the seed region. The remaining task and motion regressors were included as regressors of no interest. Significant, group-level clusters were obtained in a whole-brain analysisby conducting a 2 (Stress) x 2 (Age) x 2 (Gender) repeated measures analyses of variance (ANOVA) using the FMRIB local analysis of mixed effects (FLAME1) module in FSL with and without sleep duration (mean-centered) entered as a regressor in the model. Z (Gaussianized T) statistic images were threshold using clusters determined by $\mathrm{Z}>2.3$ and a whole-brain corrected cluster significant threshold of $\mathrm{p}<.05$ using Gaussian Random Field theory. Tests were corrected for family-wise errors (FWE). Anatomical localization within each cluster was obtained by searching within maximum likelihood regions from the FSL Harvard-Oxford probabilistic atlas.

\subsection{Statistical Analyses of Cortisol Data}

Since the time of day that participants collected salivary cortisol for baseline varied between participants, a series of linear regressions were used to individually model each participant's baseline diurnal pattern of cortisol by regressing cortisol values (Cortisol) on the time of day the cortisol sample was collected (Time) and the quadratic form of time of day $\left(\right.$ Time $\left.^{2}\right)$ across the two days of collection: Cortisol $=b_{0}+b_{1}($ Time $)+b_{2}\left(\right.$ Time $\left.^{2}\right)+$ e. One limitation in having stress states determined by participants is that the time of day that participants visited the laboratory varied. As a consequence, the time of day the salivary cortisol was collected by participants on the laboratory visits varied. One way to account for this variability in time was to first interpolate the predicted cortisol value for the time of day of the visit by substituting the time of day (in hours) into the Time and Time ${ }^{2}$ variables in each 
participant's predicted regression equation. This provided a predicted baseline cortisol value for a particular time of day for a particular participant. This predicted cortisol value was then used to calculate a difference score from the actual cortisol value collected during each laboratory visit. Three difference scores in cortisol were calculated for the three laboratory visits for each participant: Lab Cortisol Difference, High Stress Cortisol Difference, and Low Stress Cortisol Difference. These difference scores were used as measures of cortisol reactivity to stress.

\section{Results}

\subsection{Behavioral Results}

\subsubsection{Stress ratings}

A 2 (Stress) x 2 (Age) x Sleep (mean-centered) repeated measures ANCOVA, controlling for gender, was conducted on relative change in stress ratings from baselineduring high stress and low stress scans. Results revealed a main effect of stress, $F(1,39)=51.145, p<.001$ such that the difference in stress ratings relative to baseline was greater under high stress $(M=1.384)$ compared to that under low stress $(M=-.534)$. There were no significant stress $\mathrm{x}$ age $(p=.905)$, stress $\mathrm{x}$ sleep $(p=.839)$, or stress $\mathrm{x}$ sleep x age $(p=.387)$ interactions. Change in stress ratings was not correlated with change in cortisol relative to baseline for high stress or low stress conditions. Controlling for age, gender, and sleep, relative increases in stress ratings under high stress from baseline was positively correlated with overall risk-taking under high stress, averaged across frame and EV trials (partial $r=.458, p=.003$ ).

\subsubsection{Salivary cortisol}

A 2 (Stress) x 2 (Age) x Sleep (mean-centered) repeated measures ANCOVA on cortisol difference, controlling for gender and cortisol difference during the firstvisit (Lab Cortisol Difference), revealed a main effect of stress, $F(1,33)=5.527, p=.025$. Cortisol difference from 
baseline during high stress $(M=2.96 \mathrm{nmol} / \mathrm{L}, S D=8.35 \mathrm{nmol} / \mathrm{L})$ was greater than cortisol difference from baseline during low stress $(M=-.412 \mathrm{nmol} / \mathrm{L}, S D=7.78 \mathrm{nmol} / \mathrm{L})$. There were no significant stress $\mathrm{x}$ age $(p=.801)$, stress $\mathrm{x}$ sleep $(p=.382)$, or stress $\mathrm{x}$ age $\mathrm{x}$ sleep $(p=.700)$ interactions.

\subsubsection{Sleep duration \& risk-taking on the Cups task}

The main effects of stress on risky decision-making and how they were moderated by age and gender have been previously reported (Uy \& Galván, 2016).The current report focuses on how sleep duration modifies the relationship between stress and risk-taking over and above the effects of age and gender.

A 2 (Stress) x 2 (Frame) x 3 (EV) x 2 (Age) x Sleep (mean-centered) repeated measures ANCOVA, controlling for gender and scan order, revealed a main effect of $\operatorname{EV}(F(2,37)=$ 22.677, $p<.001)$, a stress $\mathrm{x}$ EV interaction $(F(2,37)=6.079, p=.005)$, and a sleep $\mathrm{x}$ stress $\mathrm{x}$ frame $x \mathrm{EV}$ interaction $(F(1,38)=5.283, p=.027)$. Interactions between sleep and stress did not differ by age; therefore, higher order interaction terms with age were removed and age was instead included as a covariate in subsequent analyses. Post-hoc simple effects were conducted to examine how sleep duration affected the relationship between stress and risky decisions, correcting for multiple comparisons using Bonferroni corrections. Analyses revealed a significant sleep $\mathrm{x}$ stress $\mathrm{x}$ frame interaction for EQEV risks $(F(1,39)=7.365, p=.010)$, but not for ADV risks $(p=.794)$ or DIS risks $(p=.195)$.The sleep x stress interaction was then tested separately for EQEV risks in the gain frame and EQEV risks in the lose frame. Analyses revealed a significant sleep $\mathrm{x}$ stress interaction for EQEV risks in the gain frame $(F(1,39)=$ 9.724, $p=.005)$, but not in the lose frame $(p=.564)$.Further analyses of the sleep $\mathrm{x}$ stress interaction for EQEV risk in the gain frame revealed that under low stress, sleep duration was 
not associated with EQEV risks in the gain frame $(p=.797)$. However, under high stress, longer sleep duration was associated with fewer EQEV risks taken in the gain frame $(t(39)=-2.822, p=$ .007) (Figure 2B).In addition, as can be observed in Figures 2C and 2F, there was a main effect of sleep duration on DIS risks such that, regardless of frame or stress state, sleep duration was negatively associated with overall DIS risks $(F(1,39)=5.698, p=.022)$. Collapsing across stress, sleep duration was not significantly associated with EQEV risks in the lose frame ( $p=$ .212) (Figure 2E). Sleep duration was also not associated with ADV risks (Figures 2A \& 2D).
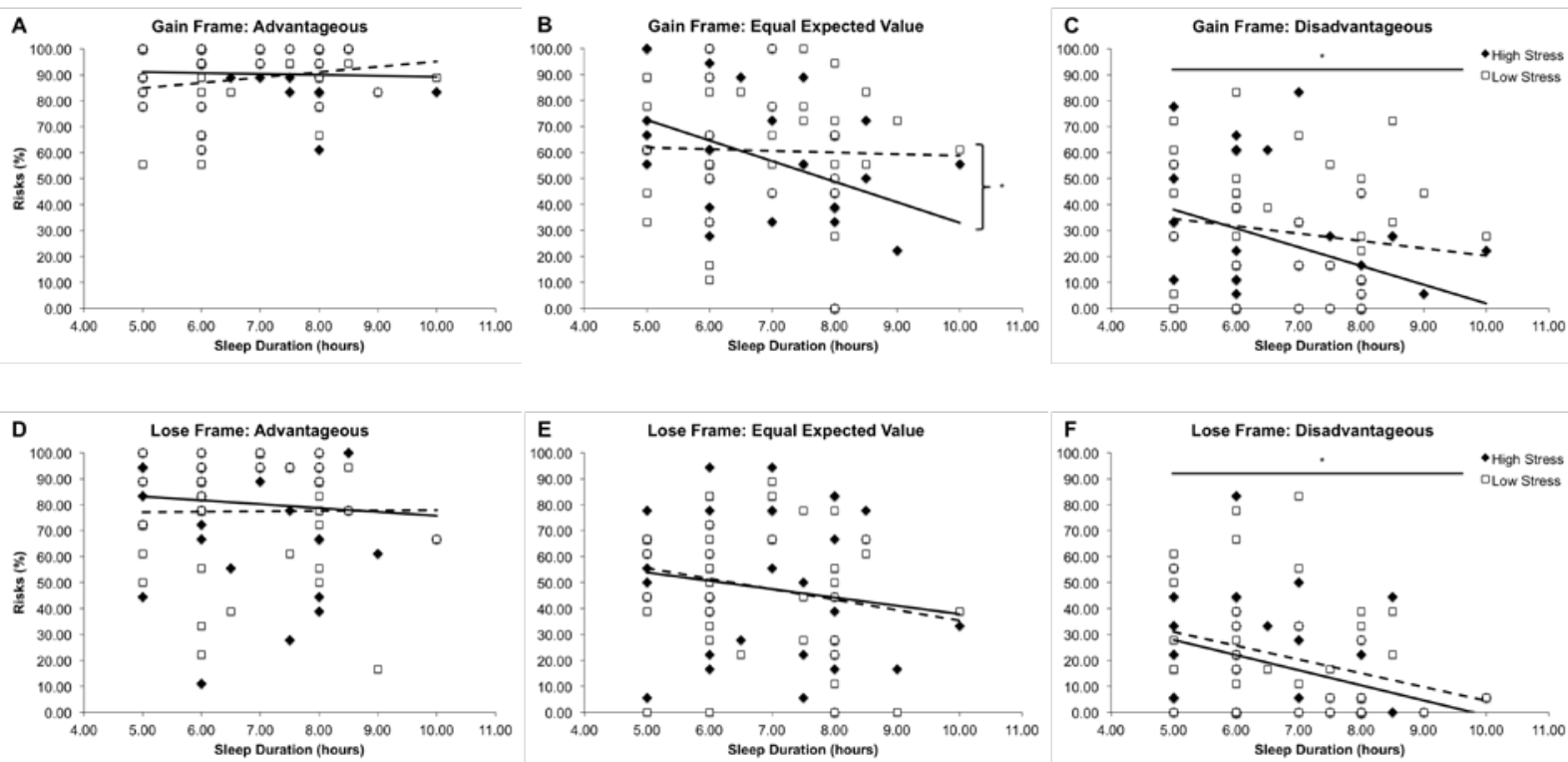

Figure 2. The relationships between sleep duration and risk-taking as a function of stress for (A) advantageous (ADV) risks in the gain frame, $(B)$ equal expected value (EQEV) risks in the gain frame, (C) disadvantageous (DIS) risks in the gain frame, (D) ADV risks in the lose frame, (E) EQEV risks in the lose frame, and (F) DIS risks in the lose frame. * denotes significant effects. 


\subsubsection{Sleep duration \& self-reported risk-taking}

A series of hierarchical linear regression analyses were conductedto examine how sleep duration relates to each subscale on the DOSPERT. Controlling for age and gender and correcting for multiple comparisons, results revealed a negative association between sleep duration and expected benefits of risk such that those who slept fewer hours per night in the past month reported greater expected benefits from all types of risks, $t(40)=-2.833, p=.007$. In addition, those who reported greater expected benefits from risks were also more likely to take those risks $(t(40)=2.824, p=.007)$. However, sleep duration was not associated with the likelihood of taking risks or perceived risk subscales on the DOSPERT. Self-reported risk-taking on the DOSPERT was not significantly associated with risk-taking on the Cups Task.

\section{2 fMRI Results}

\subsubsection{Main effect of risky choice}

ROI analysis of risky choice > safe choice contrast revealed a Stress x Age x Gender interaction in the left anterior insula (MNI coordinates: $x=-34, y=22, z=-2, Z=3.42$ ) (Figure 3A). Parameter estimates for left AI activation in the risky > safe choice contrast under high stress and low stress were extracted to determine the direction of effects. Post-hoc analyses revealed no significant group differences in left AI activation for risky> safe choice under low stress. Under high stress, however, age x gender differences emerged such that adult males engaged left AI for risky> safe to a greater extent than did adolescent males, $F(1,40)=18.497$, $p<.001$; this effect appears to be driven by a trending decrease in left AI activation for risky $>$ safe from low stress to high stress in adolescent males, $F(1,40)=3.316, p=.076$. There were no significant effects of stress on left AI activation in adolescent females $(p=.478)$ or adult females 
$(p=.913)$. There were no significant associations between sleep duration and AI activation for risky> safe choice contrast.

To determine whether left AI activation when taking risks under high stress was uniquely associated with risky behavior under high stress over and above sleep duration, a 2 (Frame) x 3 (EV) x Insula repeated measures ANCOVA, controlling for age, gender, sleep, and scan order was conducted. Results revealed an EV x Insula interaction, $F(2,37)=4.719, p=.015$. Post-hoc simple slope tests on EV trials collapsed across frame indicated that left AI activation under high stress was negatively associated with DIS risks $(t(38)=-3.196, p=.003)$ and EQEV risks $(t(38)$ $=-2.567, p=.014)$ taken under high stress, but not ADV risks under high stress $(p=.949)$. Left Alactivation under low stress was not associated with risk-taking under low stress.

\subsubsection{The interaction of sleep duration and stress-related anterior insula activation on non-} advantageous risk-taking

Given that sleep duration and AI activation appear to uniquely predict non-advantageous risk-taking under high stress, analyses were conducted to determine whether sleep duration and AI activation interact to predict risk-taking under high stress. A 3 (EV) x Insula x Sleep repeated measures ANCOVA, controlling for age, gender, and scan order, revealed an EV x insula x sleep interaction $(F(2,36)=3.918, p=.029$. Post-hoc simple effects test indicate that the insula $\mathrm{x}$ sleep interaction predicted DIS risks under high stress $(t(37)=2.206, p=.034)$, but not EQEV risks under high stress $(p=.816)$. Sleep duration groups were created to describe the nature of the interaction. Based on age and recommendations from the National Sleep Foundation, individuals were categorized into two groups: one in which sleep duration was within recommendation limits (7.0+ hours per night for adults, $n=13$; 8.0+ hours per night for adolescents, $n=7$ ) and one below recommendation limits (less than 7 hours per night for adults, 
$\mathrm{n}=9$; less than 8 hours per night for adolescents, $\mathrm{n}=15$ ). Controlling for age, gender, and scan order, simple effects tests revealed that for those sleeping below recommendation limits, left AI activation under high stress was negatively associated with DIS risks under high stress $(t(37)=$ 3.265, $p=.002$. In contrast, this relationship was attenuated for those who reported sleeping within recommendation limits $(p=.184)$ (Figure 3B). Because of the relatively larger number of adolescents sleeping below recommendation limits, it is possible that, despite statistically controlling for the effects of age, the results of the "below recommended duration" group could be driven by adolescents. As a result, sleep groups were re-categorized based on the median reported duration (7 hours). The results held: among those who reported sleeping 5.0-6.9 hours per night in the past month ( $\mathrm{n}=9$ adults, $\mathrm{n}=12$ adolescents), those who showed less activation of the left AI during risk-taking under high stress took more DIS risks under high stress, $t(36)=$ 2.408, $p=.021$, while there was still no significant relationship between left AI activation and DIS risks taken under high stress for those who slept 7.0+ hours per night in the past month $(\mathrm{n}=$ 13 adults, $\mathrm{n}=11$ adolescents), $t(36)=-.232, p=.818$.
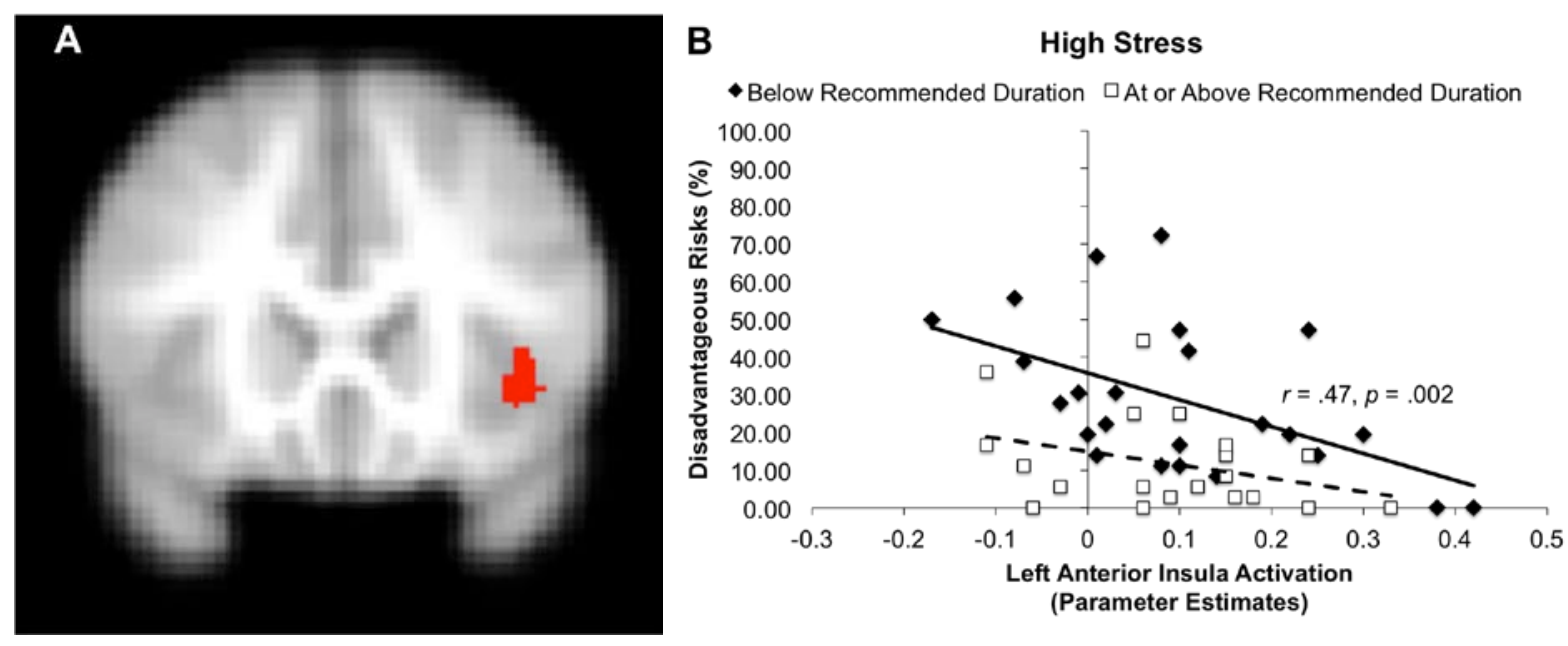
Figure 3. (A) ROI analysis of risky choice > safe choice contrast revealed a Stress x Age x

Gender interaction in the left anterior insula (MNI coordinates: $x=-34, y=22, z=-2, Z=3.42$ ):

under high stress, adult males engaged left AI for risk > safe to a greater extent than did

adolescent males, $F(1,40)=18.497, p<.001$; this effect appears to be driven by a trending

decrease in left AI activation for risky> safe from low stress to high stress in adolescent males, $F(1,40)=3.316, p=.076$. (B) Sleep $x$ Insula interaction on disadvantageous (DIS) risks taken under high stress: among those sleeping below recommendation limits, left AI activation under high stress was negatively associated with DIS risks under high stress $(t(37)=-3.265, p=.002$. In contrast, this relationship was attenuated for those who reported sleeping within recommendation limits $(p=.184)$.

\subsection{Psychophysiological Interaction Analyses}

3.3.1 Sleep duration and functional coupling between the anterior insula and prefrontal regions

The seed used for PPI analyses was defined as a $6 \mathrm{~mm}$ sphere around the peak voxel of the left AI (MNI coordinates: $x=-34, y=22, z=-2$ ). On average, during risky $>$ safe choices, left AI was functionally coupled (positively correlated) with a number of prefrontal regions, including: left DLPFC ( $x=-28, y=36, z=38, Z=3.63)$, bilateral inferior frontal gyrus (IFG), pars triangularis (right: $x=50, y=30, z=10, Z=3.53$; left: $x=-50, y=34, z=4, Z=3.47$ ), bilateral IFG, pars opercularis (right: $x=54, y=10, z=12, Z=3.4$; left: $x=-54, y=14, z=18$, $Z=3.23$ ), bilateral frontal pole (left: $x=-20, y=58, z=18, Z=4.16$; right: $x=22, y=52, z=$ $34, Z=3.42), \operatorname{vmPFC}(x=4, y=38, z=-14, Z=3.28)$, dorsomedial PFC (dmPFC; $x=-10, y=$ 58, $z=34, Z=3.58)$, and ACC $(x=4, y=34, z=4, Z=3.97)$. Left AI was also functionally 
coupled with bilateral superior temporal gyrus (left: $x=-44, y=-40, z=10, Z=3.98$, right: $x=$ 56, $y=-22, z=6, Z=3.29)$. These patterns of functional coupling did not differ as a function of stress or age.

When sleep duration was added as a regressor in the GLM, analyses revealed that, on average, sleep duration was negatively associated with functional coupling between the left AI and bilateral caudate (right: $x=10, y=6, z=22, Z=4.35$; left: $x=-12, y=10, z=22, Z=3.04$ ) (Figure 4A). This relationship did not differ as a function of stress or age. That is, for both adolescents and adults, as sleep duration increases, functional coupling strength between the left AI and bilateral caudate decreases under both high stress (Figure 4C) and low stress state (Figure 4D).Functional coupling strength between the AI and caudate was not related to risk-taking behavior.

There was a stress x sleep interaction on functional coupling between the AI and right DLPFC ( $x=26, y=34, z=38, Z=3.5)$ (Figure 4B). Parameter estimates were extracted to probe the direction of effects. Post-hoc simple effects tests, controlling for age, gender, and scan order, revealed that longer reported sleep duration was associated with greater functional coupling between the AI and right DLPFC under high stress $(t(39)=2.284, p=.028)$ (Figure $4 \mathrm{C})$, but not under low stress $(p=.078)$ (Figure 4D). Functional coupling between the AI and DLPFC was not related to risky behavior. Finally, as can be seen in Figure 4C, under high stress, those who reported sleeping longer hours showed greater divergence in functional coupling strength between AI-DLPFC and AI-caudate systems, $F(1,39)=15.653, p<.001$, while coupling strength between AI-DLPFC and AI-caudate systems did not differ as a function of sleep duration under low stress(Figure 4D). The divergence in functional coupling strength between AI-DLPFC and AI-caudate systems was not associated with risk-taking behavior. 

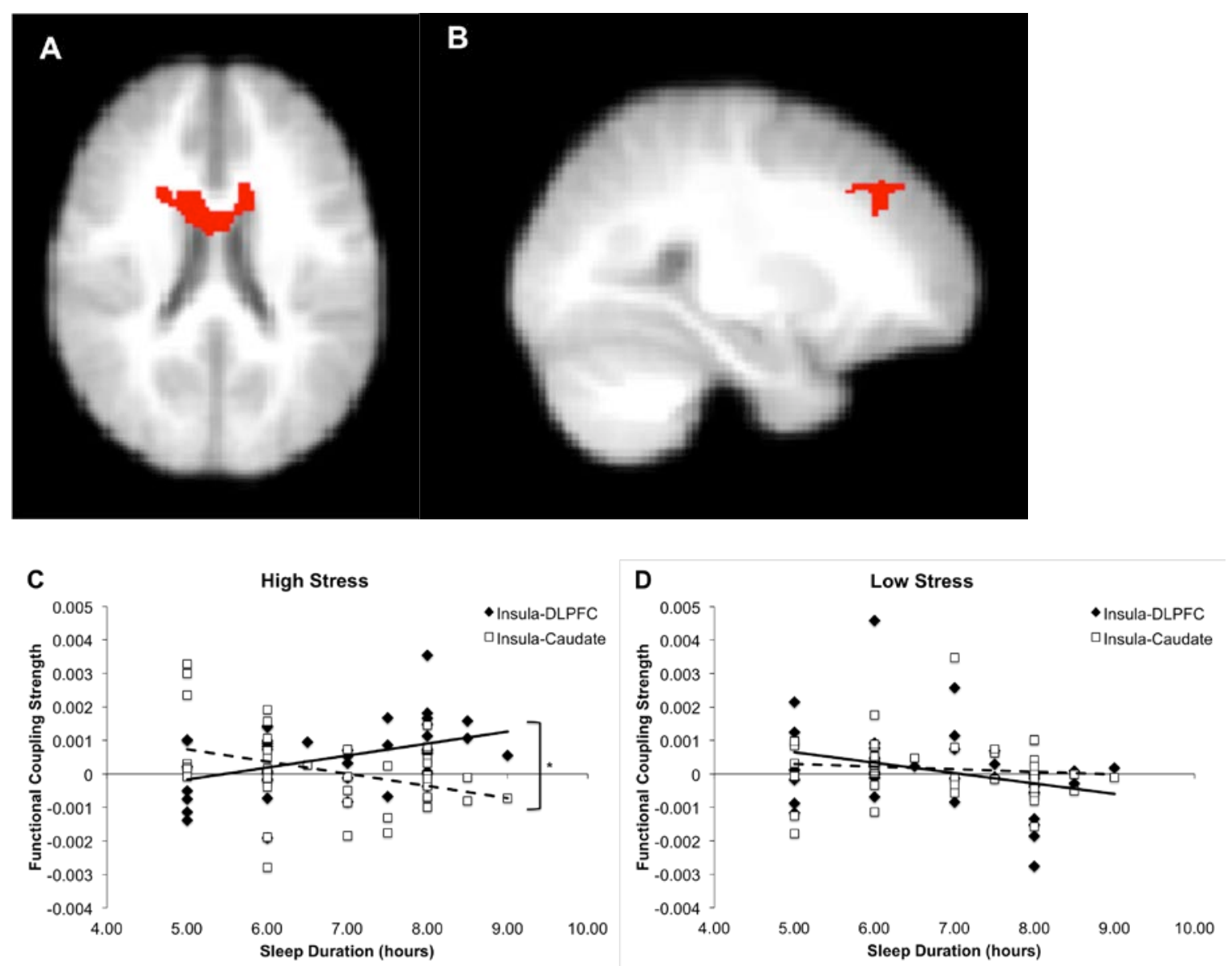

Figure 4. (A) Sleep duration was negatively associated with functional coupling between the left AI and bilateral caudate (right: $x=10, y=6, z=22, Z=4.35$; left: $x=-12, y=10, z=22, Z=$ 3.04) under both (C) high stress and (D) low stress. (B) There was a stress x sleep interaction on functional coupling between the AI and right DLPFC ( $x=26, y=34, z=38, Z=3.5)$ : longer reported sleep duration was associated with greater functional coupling between the AI and right DLPFC (C) under high stress $(t(39)=2.284, p=.028)$, but not under $(\mathrm{D})$ low stress $(p=.078)$.

\section{Discussion}


The goal of the current study was to investigate how naturalistic sleep habits affect risky decision-making and related changes in neural activity when people feel stressed. We found that sleep duration moderated the effects of stress on risky decision-making, but in somewhat unexpected ways. In particular, we predicted that shorter sleep duration would lead to greater increases in non-advantageous risks when people were experiencing high levels of stress relative to lower levels of stress (and no stress-related changes in risk-taking for those who sleep longer). Instead, as can be observed in Figure 2B, we found that those who reported longer sleep duration took fewer non-advantageous risks in the gain frame when stressed. That is, longer sleep duration was associated with fewer risky choices for decisions where the expected gain of the risky choice was not greater than that of the safe choice under stress, which could be an adaptive decision-making strategy.In contrast, there were nostress-related changes in risky behavior for those reporting fewer hours of sleep. It appears then that longer sleep duration might serve as a protective factor against stress and even allows one to reap the adaptive decision-making benefits of acute stress. These findings are consistent with previous studies reporting that sleep deprived individuals (relative to well-rested individuals) increased risk-taking for gains(Libedinsky et al., 2011; Menz et al., 2012; Mullette-Gillman et al., 2015; Venkatraman et al., 2007, 2011). Moreover, we also found that shorter sleep duration was associated with greater disadvantageous risks taken, regardless of frame or stress state, consistent with previous studies that showed that showed thatpoor sleep was related to decreases in the use of expected value information when making decisions(Libedinsky et al., 2011; Menz et al., 2012; Mullette-Gillman et al., 2015). Finally, we found that individuals who reported sleeping fewer hours per night also reported greater expected benefits from taking real world risks, corroborating the idea that individuals who obtain poor sleep may exhibita positivity bias when evaluating risks. 
We predicted that the interaction between sleep and stress on risky behavior would be stronger in adolescents relative to adults. Althoughwe did not find that the interaction was statistically significant, the effect could have been masked by the differences in sleep needs between the age groups (that adolescents might require more sleep than do adults)andthe high prevalence of adolescents not obtaining sufficient sleep inherent in the population.In other words, it could be that the effects of stress in the current study were being evaluated between sleep-deprived adolescents and well-rested adults, and that any effects of sleep observed could also reflect effects of age. Indeed, though the Chi-square test revealed no significant differences in the distribution,we haveslightly more adolescents sleeping less than their recommended 8hour minimum $(\mathrm{n}=15)$ than adults are sleeping less than their recommended 7-hour minimum (n $=9$ ). While this may be of concern for data analysis and interpretation, the distribution of our sample actually reflects the real distribution reported by the CDC. In our sample, we have about $41 \%$ of adults and $68 \%$ of adolescents who reported sleeping insufficient sleep, similar to the CDC's report of about 35\% and over 60\%, respectively.Despite being more ecologically valid, one of the drawbacks in investigating naturalistic sleep habits in adolescents is that we may not have an adequate number of adolescents getting enough sleep in our sample to adequately investigate the effects of sleep in this population. These considerations need to be taken into account wheninterpreting the results of the current study.

fMRI analyses revealed that sleep duration interacted with stress-related changes in brain activation to predict risk-taking behavior. Specifically, under high stress, individuals who showed reduced activation of the left AI during decision-making took more risks that were disadvantageous (in which participants chose a risky option that was unlikely to yield a good outcome over a safe option that would yield a small monetary gain). This relationship was 
stronger for those who reported sleeping insufficienthours (as determined by age-specific NSFrecommended duration) and was attenuated for those who reported sleeping sufficient hours, controlling for age.The results held even after re-categorizing sleep groups based on the median reported duration of 7 hours to take into account the disproportionate amount of adolescents receiving insufficient sleep (i.e., sleeping less than 8 hours per night). In other words, even when adolescents weren’t sleeping “enough”, those who reported sleeping at least a minimum of 7 hours per night on average were able to reap the same benefits as adults who obtain sufficient sleep.

Given previous literature that showed developmental difference in functional connectivity strength between the AI-affective and AI-cognitive systems, we conducted PPI analyses to determine whether the relationship between functional coupling of the AI and affective systems and functional coupling of the AI and cognitive systems was associated with risk-taking behavior and also whether functional coupling was affected by sleep duration. We found that, on average, the left AI was functionally coupled with a number of prefrontal regions including the DLPFC, IFG, vmPFC, dmPFC, and ACC, consistent with previous functional connectivity studies that investigated the AI (Fair et al., 2007; Stevens et al., 2009). This pattern of functional coupling did not differ by age, which could be due to our adolescent sample being older than those reported in previous studies (e.g., adolescents were 10-15 years of age in Fair et al., 2007). There was no main effect of stress, but there was a main effect of sleep on functional coupling between the AI and caudate, and a sleep x stress interaction in functional coupling between the AI and DLPFC. Together, under high stress, longer sleep duration was associated with decreasing functional coupling between the AI and caudate and increasing functional coupling between the AI and DLPFC when taking risks. These findings are consistent with 
previous studies that found that poor sleep was associated with reduced functional coupling between affective and regulatory regions in adults (Gujar, Yoo, Hu, \& Walker, 2011; Yoo et al., 2007), and more specifically withreduced functional coupling between the DLPFC and AI during reward processing in adolescents(Telzer et al., 2013). This divergence in functional coupling between the AI with affective and cognitive control systems under conditions of stress suggest that those who sleep longer might be better at engaging top-down regulation of affective processes during stress relative to those who sleep less, which has implications for risky behavior. In addition, that sleep duration was not associated with risky behavior or functional activity under low stress suggests that the advantages of sufficient sleep may only be evident during affective arousal. We did not find that functional coupling between the AI and either of these systems or the extent to which divergence in functional coupling between the AI and these two systems under high stress was associated with risky behavior. However, these fMRI findings,in conjunction with more optimal behavior in the gain frame under high stress for those who sleep longer suggest that this could be one potential (perhaps indirect) mechanism by which achieving sufficient sleep confers advantages in the face of stress.

Decision-making behaviors are sensitive to changes in dopaminergic activity, which are influenced by both acute stress and sleep loss, providing a possible mechanism that explains how stress and habitual sleep loss affect decision-making. In rats, increases in mRNA expression of D1 receptors in the nucleus accumbens and insular cortex were positively associated with risktaking to large rewards(Simon et al., 2011), indicating that D1 receptors in the insula may be involved in valuation of risky versus safe outcomes and cost-benefit decision-making(Cardinal, 2006; Naqvi \& Bechara, 2009). In humans, genetic polymorphisms associated with high levels of D1 receptors in the striatum were predictive of greater reward-driven behavior during decision- 
making(Frank, Moustafa, Haughey, Curran, \& Hutchison, 2007; Richard \& Berridge, 2011). Acute stress influences risky decision-making behavior through cortisol-related increases in dopamine release in the striatum and orbitofrontal cortex(Pruessner et al., 2004; Ungless et al., 2010), which have bidirectional projections with the insula(Mesulam \& Mufson, 1982; Mufson \& Mesulam, 1982), potentially influencing D1 receptor activity in the insula through stressrelated changes in the dopaminergic system. Animal models demonstrate that glucocorticoidrelated increase in dopamine release on the nucleus accumbens and PFC increases D1 receptor binding in the PFC to the point of which impairs cognitive function(Ambroggi et al., 2009; Butts, Weinberg, Young, \& Phillips, 2011; Murphy, Arnsten, Goldman-Rakic, \& Roth, 1996; Vijayraghavan, Wang, Birnbaum, Williams, \& Arnsten, 2007) and impacts optimal regulation of subcortical structures (Arnsten \& Goldman-Rakic, 1998; Raio, Orederu, Palazzolo, Shurick, \& Phelps, 2013). These findings suggest that stress may act specifically on D1 receptors.

On the other hand, activation of D2 receptors in the striatum via systemic increases in D2 agonists in rats attenuated preferences for large, risky rewards as the risk of punishment increased, underlying risk-avoidant behaviors(Simon et al., 2011). In humans, genetic polymorphisms associated with high D2 receptors in the striatum were associated with greater avoidance of punishment(Frank et al., 2007; Richard \& Berridge, 2011). Sleep deprivation affects the dopaminergic system by possibly up-regulating concentrations of dopamine to maintain wakefulness(Volkow et al., 2008, 2009) while also down-regulating D2 receptor availability in the ventral striatum(Volkow et al., 2012), which has implications for risky decision-making behavior. Taken together, stress and sleep deprivation might concomitantly increase D1 receptor binding and decrease D2 receptor binding, respectively, to alter decisionmaking behavior. These relationships, however, are yet to be experimentally tested. Using fMRI, 
researchers have suggested that enhanced ventral striatal reactivity to monetary gains and attenuated insula activity to losses during risk-taking following sleep deprivation may reflect dopaminergic changes(Venkatraman et al., 2007, 2011). Though plausible, dopamine was not directly measured in those studies or in the current study, precluding a definitive conclusion. Future research utilizing animal models and PET studies in humans could provide insight into the processes by which sleep loss and acute stress interact to modify risky decision-making behavior.

The results of the current study are novel as they are the first to characterize how normative sleep duration affects risky decision-making when adolescents and adults experience naturally occurring daily stress. Nevertheless, there are a few limitations to note. First, given that there were no significant differences in sleep duration between the two groups and that adolescents require more sleep than adults, our sample may have included more adolescents than adults who experienced poor sleep. However, a Chi-square test revealed no significant differences in the distribution of individuals who reported receiving insufficient sleep between adolescents and adults. Future studies investigating adolescents should be mindful of this limitation during sampling and attempt to also enroll adolescents who are getting enough sleep. Second, our measure of sleep duration was determined by participants’ self-reported sleep duration. Self-reported sleep duration has been shown to be moderately correlated $(r=0.45)$ with objective sleep measures obtained using actigraph watches and daily diary in adults(Biddle et al., 2015; Cespedes et al., 2016; Lauderdale, 2008) and in adolescents(Short et al., 2012; Wolfson et al., 2003). If there were any biases, self-reported measures of habitual sleep duration tended to overestimate actual sleep duration (Biddle et al., 2015; Cespedes et al., 2016; Lauderdale, 2008), raising the possibility that our participants could have been actually sleeping fewer hours per 
night than they reported. If these biases were true in our sample (i.e., if participants were sleeper fewer hours than they reported), then the current study is actually a conservative report of how sleep impacts the association between neural activation and risky decision making. Perhaps using more objective measures of sleep duration would yield stronger effects of sleep on stress-related neural changes and behavior. Alternatively, it is also possible that participants' subjective reports of how much sleep they receive could modify their appraisals of stressors and subsequent behaviors to a greater extent than would objective reports of sleep. Third, self-reported sleep duration was only measured when participants enrolled in the study. We did not collect measures of sleep at each visit. It is possible that participants who reported long habitual sleep duration at the beginning of the study could have slept poorly the night before, or vice-versa. On the other hand, we speculate that what participants reported at the beginning of the study is consistent with what they would have reported during the stress visits.To arrive at a more definitive conclusion, future studies should track daily diary reports of sleep habits in conjunction with objective sleep reports using actigraph watches and daily stress ratings to further explore the relationships between daily sleep and stress. Fourth, the non-experimental nature of our stress induction limits our ability to precisely characterize the role of cortisol in stress-related differences in neural response. Nonetheless, our results suggest that our ecologically-valid stress assessment is robust and captures stress-related differences in the neural correlates of risky decision-making behavior. Additionally, the non-experimental nature of our study precludes any claims about causality. However, researchers have found that individuals who experienced just one night without sleep showed greater baseline cortisol and greater cortisol response to acute stress than those who received sufficient sleep (Minkel et al., 2014), suggesting a causal link between sleep and HPA axis functioning. The current study did not find that naturalistic sleep duration was related to 
cortisol reactivity to stress, which could be due to our approximate measure of sleep duration, the delayed measure of cortisol relative to stressor onset, or might suggest that more extreme forms of sleep loss may be necessary to detect observable changes in HPA functioning.Finally, it is possible that inexperienced MRI participants mightfind the fMRI experience stressful, which could conflate the fMRI data collected on the different stress days.Consequently, we controlled for scan order in our analyses to account for this possibility and the results held. Though we did not collect data on participants' scanner experience in the current study, weprepared participants for the fMRI environment using a simulated scanner and followed recommendations suggested by Galván, Van Leijenhorst, and McGlennen (2012) to acquire meaningful and informative fMRI data.

In sum, our findings highlight the neural costs of habitually attaining insufficient sleep by showing that even normative amounts of shorter sleep duration can interact with stress- and riskrelated neural regions and lead to suboptimal decision-making in stressful contexts. 


\section{References}

Almeida, D. M., McGonagle, K., \& King, H. (2009). Assessing daily stress processes in social surveys by combining stressor exposure and salivary cortisol. Biodemography and Social Biology, 55(2), 219-237. http://doi.org/10.1080/19485560903382338

Ambroggi, F., Turiault, M., Milet, A., Deroche-Gamonet, V., Parnaudeau, S., Balado, E., ... Tronche, F. (2009). Stress and addiction: glucocorticoid receptor in dopaminoceptive neurons facilitates cocaine seeking. Nature Neuroscience, 12(3), 247-249. http://doi.org/10.1038/nn.2282

Anderson, C., \& Platten, C. R. (2011). Sleep deprivation lowers inhibition and enhances impulsivity to negative stimuli. Behavioural Brain Research, 217(2), 463-466. http://doi.org/10.1016/j.bbr.2010.09.020

Arnsten, A. F., \& Goldman-Rakic, P. S. (1998). Noise stress impairs prefrontal cortical cognitive function in monkeys: evidence for a hyperdopaminergic mechanism. Archives of General Psychiatry, 55(4), 362-368. http://doi.org/10.1001/archpsyc.55.4.362

Balbo, M., Leproult, R., \& Van Cauter, E. (2010). Impact of sleep and its disturbances on hypothalamo-pituitary-adrenal axis activity. International Journal of Endocrinology, 2010, 1-16. http://doi.org/10.1155/2010/759234

Bechara, A., \& Damasio, A. R. (2005). The somatic marker hypothesis: A neural theory of economic decision. Games and Economic Behavior, 52(2), 336-372. http://doi.org/10.1016/j.geb.2004.06.010

Bechara, A., Damasio, H., Tranel, D., \& Damasio, A. R. (1997). Deciding advantageously before knowing the advantageous strategy. Science (New York, N.Y.), 275(5304), 1293-1295. http://doi.org/10.1126/science.275.5304.1293 
Biddle, D. J., Robillard, R., Hermens, D. F., Hickie, I. B., \& Glozier, N. (2015). Accuracy of self-reported sleep parameters compared with actigraphy in young people with mental illhealth. Sleep Health, 1(3), 214-220. http://doi.org/10.1016/j.sleh.2015.07.006

Bolger, N., Davis, A., \& Rafaeli, E. (2003). Diary methods: Capturing life as it is lived. Annual Review of Psychology, 54(1), 579-616. http://doi.org/10.1146/annurev.psych.54.101601.145030

Butts, K. A., Weinberg, J., Young, A. H., \& Phillips, A. G. (2011). Glucocorticoid receptors in the prefrontal cortex regulate stress-evoked dopamine efflux and aspects of executive function. Proceedings of the National Academy of Sciences, 108(45), 18459-18464. http://doi.org/10.1073/pnas.1111746108

Cardinal, R. N. (2006). Neural systems implicated in delayed and probabilistic reinforcement. Neural Networks, 19(8), 1277-1301. http://doi.org/10.1016/j.neunet.2006.03.004 CDC. (2011). Unhealthy sleep-related behaviors--12 States, 2009. MMWR. Morbidity and Mortality Weekly Report, 60(8), 233-238.

Cespedes, E. M., Hu, F. B., Redline, S., Rosner, B., Alcantara, C., Cai, J., ... Patel, S. R. (2016). Comparison of Self-Reported Sleep Duration with Actigraphy: Results from the Hispanic Community Health Study/Study of Latinos Sue??o Ancillary Study. American Journal of Epidemiology, 183(6), 561-573. http://doi.org/10.1093/aje/kwv251

Droutman, V., Bechara, A., \& Read, S. J. (2015). Roles of the different sub-regions of the insular cortex in various phases of the decision-making process. Frontiers in Behavioral Neuroscience, 9(November), 1-14. http://doi.org/10.3389/fnbeh.2015.00309

Eshel, N., Nelson, E. E., Blair, J., Pine, D. S., \& Ernst, M. (2007). Neural substrates of choice selection in adults and adolescents: development of the ventrolateral prefrontal and anterior 
cingulate cortices. Neuropsychologia, 45(6), 1270-1279.

http://doi.org/10.1016/j.neuropsychologia.2006.10.004.Neural

Fair, D. A., Dosenbach, N. U. F., Church, J. A., Cohen, A. L., Brahmbhatt, S., Miezin, F. M., ... Schlaggar, B. L. (2007). Development of distinct control networks through segregation and integration. Proceedings of the National Academy of Sciences of the United States of America, 104(33), 13507-12. http://doi.org/10.1073/pnas.0705843104

Figner, B., \& Weber, E. U. (2012). Who takes risks when and why? Determinants of risk taking. Current Directions in Psychological Science, 20(4), 211-216. http://doi.org/10.2139/ssrn.1441273

Frank, M. J., Moustafa, A. A., Haughey, H. M., Curran, T., \& Hutchison, K. E. (2007). Genetic triple dissociation reveals multiple roles for dopamine in reinforcement learning. Proceedings of the National Academy of Sciences of the United States of America, 104(41), 16311-16316. http://doi.org/10.1073/pnas.0706111104

Friston, K. J., Buechel, C., Fink, G. R., Morris, J., Rolls, E., \& Dolan, R. J. (1997). Psychophysiological and modulatory interactions in neuroimaging. Neuroimage, 6(3), 218229. http://doi.org/10.1006/nimg.1997.0291

Galván, A., \& McGlennen, K. M. (2012). Daily stress increases risky decision-making in adolescents: a preliminary study. Developmental Psychobiology, 54(4), 433-40. http://doi.org/10.1002/dev.20602

Galván, A., \& Rahdar, A. (2013). The neurobiological effects of stress on adolescent decision making. Neuroscience, 249, 223-31. http://doi.org/10.1016/j.neuroscience.2012.09.074

Galván, A., Van Leijenhorst, L., \& McGlennen, K. M. (2012). Considerations for imaging the adolescent brain. Developmental Cognitive Neuroscience, 2(3), 293-302. 
http://doi.org/10.1016/j.dcn.2012.02.002

Gujar, N., Yoo, S.-S., Hu, P., \& Walker, M. P. (2011). Sleep Deprivation Amplifies Reactivity of Brain Reward Networks, Biasing the Appraisal of Positive Emotional Experiences. Journal of Neuroscience, 31(12), 4466-4474. http://doi.org/10.1523/JNEUROSCI.3220-10.2011

Harrison, Y., \& Horne, J. A. (2000). The impact of sleep deprivation on decision making: A review. Journal of Pxperimental Psychology. Applied, 6(3), 236-249. http://doi.org/10.1037/1076-898X.6.3.236

Hellhammer, D. H., Wüst, S., \& Kudielka, B. M. (2009). Salivary cortisol as a biomarker in stress research. Psychoneuroendocrinology, 34(2), 163-171. http://doi.org/10.1016/j.psyneuen.2008.10.026

Hirshkowitz, M., Whiton, K., Albert, S. M., Alessi, C., Bruni, O., DonCarlos, L., ... Adams Hillard, P. J. (2015). National Sleep Foundation's sleep time duration recommendations: methodology and results summary. Sleep Health, 1(1), 40-43. http://doi.org/10.1016/j.sleh.2014.12.010

Kann, L., Kinchen, S., Shanklin, S. L., Flint, K. H., Kawkins, J., Harris, W. a, ... Zaza, S. (2014). Youth risk behavior surveillance--United States, 2013. Morbidity and Mortality Weekly Report. Surveillance Summaries (Washington, D.C. : 2002), 63 Suppl 4(4), 1-168. http://doi.org/ss6304a1 [pii]

Killgore, W. D. S., Balkin, T. J., \& Wesensten, N. J. (2006). Impaired decision making following 49 h of sleep deprivation. Journal of Sleep Research, 15(1), 7-13. http://doi.org/10.1111/j.1365-2869.2006.00487.x

Killgore, W. D. S., Kamimori, G. H., \& Balkin, T. J. (2011). Caffeine protects against increased risk-taking propensity during severe sleep deprivation. Journal of Sleep Research, 
20(January 2016), 395-403. http://doi.org/10.1111/j.1365-2869.2010.00893.x

Kogler, L., Mueller, V. I., Chang, A., Eickhoff, S. B., Gur, R. C., \& Derntl, B. (2013).

Psychosocial vs . physiological - an ALE meta-analysis on the neural correlates of stress reactions. NeuroImage, 119, 1328. http://doi.org/10.1016/j.neuroimage.2015.06.059

Lauderdale, D. (2008). Sleep duration: how well do self-reports reflect objective measures? The CARDIA Sleep Study. Epidemiology, 19(6), 838-45. http://doi.org/10.1097/EDE.0b013e318187a7b0.Sleep

Levin, I. P., \& Hart, S. S. (2003). Risk preferences in young children: Early evidence of individual differences in reaction to potential gains and losses. Journal of Behavioral Decision Making, 16(October), 397-413. http://doi.org/10.1002/bdm.453

Levin, I. P., Weller, J. A., Pederson, A. A., \& Harshman, L. A. (2007). Age-related differences in adaptive decision making: Sensitivity to expected value in risky choice. Judgment and Decision Making, 2(4), 225-233. Retrieved from http://journal.sjdm.org/7404/jdm7404.htm Libedinsky, C., Smith, D. V., Teng, C. S., Namburi, P., Chen, V. W., Huettel, S. a., \& Chee, M. W. L. (2011). Sleep Deprivation Alters Valuation Signals in the Ventromedial Prefrontal Cortex. Frontiers in Behavioral Neuroscience, 5(October), 1-10. http://doi.org/10.3389/fnbeh.2011.00070

Lighthall, N. R., Mather, M., \& Gorlick, M. a. (2009). Acute stress increases sex differences in risk seeking in the balloon analogue risk task. PloS One, 4(7), e6002. http://doi.org/10.1371/journal.pone.0006002

Lighthall, N. R., Sakaki, M., Vasunilashorn, S., Nga, L., Somayajula, S., Chen, E. Y., ... Mather, M. (2012). Gender differences in reward-related decision processing under stress. Social Cognitive and Affective Neuroscience, 7(4), 476-84. http://doi.org/10.1093/scan/nsr026 
Menz, M. M., Buchel, C., \& Peters, J. (2012). Sleep deprivation is associated with attenuated parametric valuation and control signals in the midbrain during value-based decision making. Journal of Neuroscience, 32(20), 6937-6946. http://doi.org/10.1523/JNEUROSCI.3553-11.2012

Mesulam, M. M., \& Mufson, E. J. (1982). Insula of the old world monkey. III: Efferent cortical output and comments on function. The Journal of Comparative Neurology, 212(1), 38-52. http://doi.org/10.1002/cne.902120104

Minkel, J., Moreta, M., Muto, J., Htaik, O., Jones, C., Basner, M., \& Dinges, D. (2014). Sleep deprivation potentiates HPA axis stress reactivity in healthy adults. Health Psychology, 33(11), 1430-1434. http://doi.org/10.1037/a0034219

Mizuhiki, T., Richmond, B. J., \& Shidara, M. (2012). Encoding of reward expectation by monkey anterior insular neurons. Journal of Neurophysiology, 107(11), 2996-3007. http://doi.org/10.1152/jn.00282.2011

Mufson, E. J., \& Mesulam, M. M. (1982). Insula of the old world monkey. II: Afferent cortical input and comments on the claustrum. The Journal of Comparative Neurology, 212, 23-37. http://doi.org/10.1002/cne.902120104

Mullette-Gillman, O. A., Kurnianingsih, Y. A., \& Liu, J. C. J. (2015). Sleep deprivation alters choice strategy without altering uncertainty or loss aversion preferences. Frontiers in Neuroscience, 9(October), 1-12. http://doi.org/10.3389/fnins.2015.00352

Murphy, B. L., Arnsten, A. F. T., Goldman-Rakic, P. S., \& Roth, R. H. (1996). Increased dopamine turnover in the prefrontal cortex impairs spatial working memory performance in rats and monkeys. Proceedings of the National Academy of Sciences of the United States of America, 93(February), 1325-1329. 
Naqvi, N. H., \& Bechara, A. (2009). The hidden island of addiction: the insula. Trends in Neurosciences, 32(1), 56-67. http://doi.org/10.1016/j.tins.2008.09.009

Porcelli, A. J., \& Delgado, M. R. (2009). Acute stress modulates risk taking in financial decision making. Psychological Science, 20(3), 278-283. http://doi.org/10.1111/j.14679280.2009.02288.x

Preuschoff, K., Quartz, S. R., \& Bossaerts, P. (2008). Human insula activation reflects risk prediction errors as well as risk. Journal of Neuroscience, 28(11), 2745-2752. http://doi.org/10.1523/JNEUROSCI.4286-07.2008

Pruessner, J. C., Champagne, F., Meaney, M. J., \& Dagher, A. (2004). Dopamine release in response to a psychological stress in humans and its relationship to early life maternal care: a positron emission tomography study using [11C]raclopride. The Journal of Neuroscience : The Official Journal of the Society for Neuroscience, 24(11), 2825-2831. http://doi.org/10.1523/JNEUROSCI.3422-03.2004

Pruessner, J. C., Dedovic, K., Khalili-Mahani, N., Engert, V., Pruessner, M., Buss, C., ... Lupien, S. (2008). Deactivation of the limbic system during acute psychosocial stress: Evidence from positron emission tomography and functional magnetic resonance imaging studies. Biological Psychiatry, 63(2), 234-240. http://doi.org/10.1016/j.biopsych.2007.04.041

Putman, P., Antypa, N., Crysovergi, P., \& van der Does, W. A. J. (2010). Exogenous cortisol acutely influences motivated decision making in healthy young men. Psychopharmacology, 208(2), 257-63. http://doi.org/10.1007/s00213-009-1725-y

Raio, C. M., Orederu, T. A., Palazzolo, L., Shurick, A. A., \& Phelps, E. A. (2013). Cognitive emotion regulation fails the stress test. Proceedings of the National Academy of Sciences of 
the United States of America, 110(37), 15139-44. http://doi.org/10.1073/pnas.1305706110

Richard, J. M., \& Berridge, K. C. (2011). Nucleus accumbens dopamine/glutamate interaction switches modes to generate desire versus dread: D1 alone for appetitive eating but D1 and D2 together for fear. Journal of Neuroscience, 31(36), 12866-12879. http://doi.org/10.1523/JNEUROSCI.1339-11.2011

Shaw, P., Kabani, N. J., Lerch, J. P., Eckstrand, K., Lenroot, R., Gogtay, N., ... Wise, S. P. (2008). Neurodevelopmental trajectories of the human cerebral cortex. Journal of Neuroscience, 28(14), 3586-94. http://doi.org/10.1523/JNEUROSCI.5309-07.2008

Short, M. A., Gradisar, M., Lack, L. C., Wright, H., \& Carskadon, M. A. (2012). The discrepancy between actigraphic and sleep diary measures of sleep in adolescents. Sleep Medicine, 13(4), 378-384. http://doi.org/10.1016/j.sleep.2011.11.005

Simon, N. W., Montgomery, K. S., Beas, B. S., Mitchell, M. R., LaSarge, C. L., Mendez, I. A., ... Setlow, B. (2011). Dopaminergic modulation of risky decision-making. Journal of Neuroscience, 31(48), 17460-17470. http://doi.org/10.1523/JNEUROSCI.3772-11.2011

Smith, A. R., Steinberg, L., \& Chein, J. (2014). The role of the anterior insula in adolescent decision making. Developmental Neuroscience, 196-209. http://doi.org/10.1159/000358918

Spiegel, K., Leproult, R., \& Van Cauter, E. (1999). Impact of sleep debt on metabolic and endocrine function. Lancet, 354(9188), 1435-1439. http://doi.org/10.1016/S01406736(99)01376-8

Starcke, K., \& Brand, M. (2012). Decision making under stress: a selective review. Neuroscience and Biobehavioral Reviews, 36(4), 1228-48. http://doi.org/10.1016/j.neubiorev.2012.02.003

Starcke, K., Wolf, O. T., Markowitsch, H. J., \& Brand, M. (2008). Anticipatory stress influences decision making under explicit risk conditions. Behavioral Neuroscience, 122(6), 1352- 
1360. http://doi.org/10.1037/a0013281

Stevens, M. C., Pearlson, G. D., \& Calhoun, V. D. (2009). Changes in the interaction of restingstate neural networks from adolescence to adulthood. Human Brain Mapping, 30(8), 23562366. http://doi.org/10.1002/hbm.20673

Strahler, J., Mueller, A., Rosenloecher, F., Kirschbaum, C., \& Rohleder, N. (2010). Salivary $\alpha$ amylase stress reactivity across different age groups. Psychophysiology, 47(3), 587-595. http://doi.org/10.1111/j.1469-8986.2009.00957.x

Telzer, E. H., Fuligni, A. J., Lieberman, M. D., \& Galván, A. (2013). The effects of poor quality sleep on brain function and risk taking in adolescence. NeuroImage, 71, 275-283. http://doi.org/10.1016/j.neuroimage.2013.01.025

Ungless, M. A., Argilli, E., \& Bonci, A. (2010). Effects of stress and aversion on dopamine neurons: Implications for addiction. Neuroscience and Biobehavioral Reviews, 35(2), 151156. http://doi.org/10.1016/j.neubiorev.2010.04.006

Uy, J. P., \& Galván, A. (2016). Acute stress increases risky decisions and dampens prefrontal activation among adolescent boys. NeuroImage. http://doi.org/10.1016/j.neuroimage.2016.08.067

Venkatraman, V., Chuah, Y. M. L., Huettel, S. A., \& Chee, M. W. L. (2007). Sleep deprivation elevates expectation of gains and attenuates response to losses following risky decisions. Sleep, 30(5), 603-609.

Venkatraman, V., Huettel, S. A., Chuah, L. Y. M., Payne, J. W., \& Chee, M. W. L. (2011). Sleep Deprivation Biases the Neural Mechanisms Underlying Economic Preferences. Journal of Neuroscience, 31(10), 3712-3718. http://doi.org/10.1523/JNEUROSCI.4407-10.2011 Vgontzas, A. N., Zoumakis, E., Bixler, E. O., Lin, H.-M., Follett, H., Kales, A., \& Chrousos, G. 
P. (2004). Adverse effects of modest sleep restriction on sleepiness, performance, and inflammatory cytokines. The Journal of Clinical Endocrinology and Metabolism, 89(5), 2119-26. http://doi.org/10.1210/jc.2003-031562

Vijayraghavan, S., Wang, M., Birnbaum, S. G., Williams, G. V, \& Arnsten, A. F. T. (2007). Inverted-U dopamine D1 receptor actions on prefrontal neurons engaged in working memory. Nature Neuroscience, 10(3), 376-384. http://doi.org/10.1038/nn1846

Volkow, N. D., Tomasi, D., Wang, G.-J., Telang, F., Fowler, J. S., Logan, J., ... Ferré, S. (2012). Evidence that sleep deprivation downregulates dopamine D2R in ventral striatum in the human brain. Journal of Neuroscience, 32(19), 6711-6717. http://doi.org/10.1523/JNEUROSCI.0045-12.2012

Volkow, N. D., Tomasi, D., Wang, G., Telang, F., Fowler, J. S., Wang, R. L., ... James, M. (2009). Hyperstimulation of striatal D2 receptors with sleep deprivation: Implications for cognitive impairment. NeuroImage, 45(4), 1232-1240. http://doi.org/10.1016/j.neuroimage.2009.01.003.Hyperstimulation

Volkow, N. D., Wang, G., Telang, F., Fowler, J. S., Logan, J., Wong, C., ... Jayne, M. (2008). Sleep deprivation decreases [11C] racloprides’s binding to dopamine D2/D3 receptors in the human brain. Journal of Neuroscience, 28(34), 8454-8461. http://doi.org/10.1523/JNEUROSCI.1443-08.2008.Sleep

Walker, M. P., \& Stickgold, R. (2006). Sleep, memory, and plasticity. Annual Review of Psychology, 57, 139-166. http://doi.org/10.1146/annurev.psych.56.091103.070307

Weller, J. a, Levin, I. P., Shiv, B., \& Bechara, A. (2009). The effects of insula damage on decision-making for risky gains and losses. Social Neuroscience, 4(April 2014), 347-358. http://doi.org/10.1080/17470910902934400 
Werner, N. S., Schweitzer, N., Meindl, T., Duschek, S., Kambeitz, J., \& Schandry, R. (2013). Interoceptive awareness moderates neural activity during decision-making. Biological Psychology, 94(3), 498-506. http://doi.org/10.1016/j.biopsycho.2013.09.002

Wolfson, A. R., Carskadon, M. a, Acebo, C., Seifer, R., Fallone, G., Labyak, S. E., \& Martin, J. L. (2003). Evidence for the validity of a sleep habits survey for adolescents. Sleep, 26(2), 213-216.

Xue, G., Lu, Z., Levin, I. P., \& Bechara, A. (2011). The impact of prior risk experiences on subsequent risky decision-making: The role of the insula. NeuroImage, 50(2), 709-716. http://doi.org/10.1016/j.neuroimage.2009.12.097.The

Xue, G., Lu, Z., Levin, I. P., \& Bechara, A. (2013). An fMRI study of risk-taking following wins and losses: Implications for the gambler’s fallacy. Human Brain Mapping, 18(9), 11991216. http://doi.org/10.1002/hbm.21015

Xue, G., Lu, Z., Levin, I. P., Weller, J. A., Li, X., \& Bechara, A. (2009). Functional dissociations of risk and reward processing in the medial prefrontal cortex. Cerebral Cortex (New York, N.Y. : 1991), 19(5), 1019-27. http://doi.org/10.1093/cercor/bhn147

Yoo, S. S., Gujar, N., Hu, P., Jolesz, F. A., \& Walker, M. P. (2007). The human emotional brain without sleep - a prefrontal amygdala disconnect. Current Biology, 17(20), 877-878. http://doi.org/10.1016/j.cub.2007.08.007 